\title{
As a healthcare professional, are you checking all the vital signs to evaluate total patient condition?
}

\section{Opinion}

For many years, four vital signs were used to determine the condition of a patient. Today, modern health care professionals are checking two more vital signs, pain and emotional distress.

Six vital signs are now recognized by many healthcare professionals to determine a patient's total condition:

1. Body Temperature

2. Heart Pulse Rate

3. Blood Pressure

4. Respiratory Rate

5. Pain

6. Emotional Distress

The first four are routinely measured, either manually by a person or automatically by a medical monitor. The last two, pain and distress, are new vital signs and are determined by patient interview. Pain is determined by asking the patient to rate their pain using a pain scale. Similarly, emotional distress is determined by asking the patient to complete a questionnaire.

It might be said that pain and emotional distress could be combined, since a patient in pain is also in distress. Conversely, when a person is stressed the perception of pain is greater.

\section{Wouldn't it be helpful if pain and emotional distress could be measured quantitatively just like the other vital signs?}

Perhaps they can. Biofeedback devices, such as galvanic skin response sensors, could measure and record the ongoing distress situation within a patient. Biofeedback has been used to for years to evaluate stress also called distress. There are many forms of equipment and devices available, some even for home use. Here are some points to ponder:

1. It is well known that inflicting pain, e.g. skin pinching, produces a strong biofeedback response. Therefore, perhaps, biofeedback could be used to measure and track pain. Detecting pain in infants is one example of where biofeedback is now used to detect pain and distress.

2. It is also well known that emotional situations, e.g. being frightened of medical procedures, can also produce a strong biofeedback response.

3. Lastly, it is also well known that a relaxed patient is easier to treat and will heal better and faster.

Therefore, doesn't it make sense to include biofeedback sensors along with the usual thermometer, stethoscope, and sphygmomanometer?

Taking this concept one step further, the bedside medical monitor
Volume 9 Issue 5 - 2018

\author{
Tom Garz \\ Writer and Inventor,TG Ideas LLC, USA
}

Correspondence: Tom Garz,Writer and Inventor,TG Ideas LLC, USA, Email tgideas@gmail.com

Received: May 28, 2018| Published: October 10, 2018

is already measuring all the information necessary from which to calculate the emotional distress or pain level.

\section{Perhaps, future medical monitors will have an emotional stress (distress) indicator.}

Takeaways:

1. All six vital signs should be measured to determine the total condition of a patient.

2. Pain and emotional distress can be measured and recorded just like the other vital signs.

3. Biofeedback devices could be one way to track pain and emotional distress. ${ }^{1-7}$

\section{Acknowledgement}

None.

\section{Conflict of interest}

The author declares that there is no conflict of interest.

\section{References}

1. Barry D Bultz, Linda E Carlson. Emotional distress: the sixth vital signfuture directions in cancer care. Psycho-Oncology. 2006;15(2):93-95.

2. Medical Monitor with an Emotional Stress Indicator - an Idea Ahead of Its Time. 2014

3. Guide to Implementing Screening for Distress, the $6^{\text {th }}$ Vital Sign.

4. Vital Signs.

5. Pain as the $5^{\text {th }}$ Vital Sign Toolkit. 2000.

6. Munsters J1, Wallström L, Agren J. Skin conductance measurements as pain assessment in newborn infants born at 22-27 weeks gestational age at different postnatal age. Early Hum Dev. 2012;88(1):21-26.

7. Susanne Babbel. The Connections Between Emotional Stress, Trauma and Physical Pain. Psychology Today. 2010. 